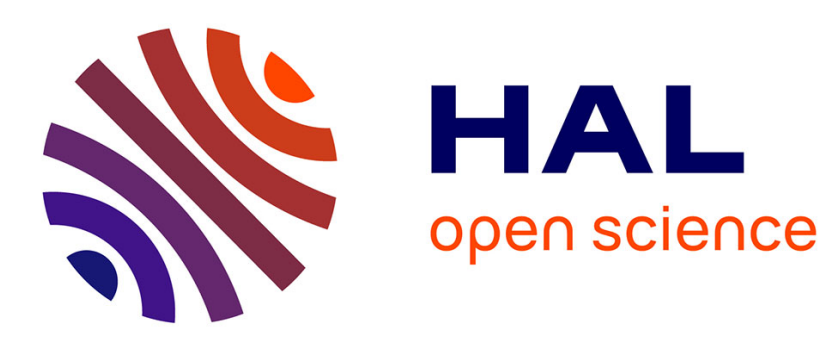

\title{
A derivative free optimization method for reservoir characterization inverse problem
}

Hoël Langouët, Delphine Sinoquet, Sébastien da Veiga

\section{To cite this version:}

Hoël Langouët, Delphine Sinoquet, Sébastien da Veiga. A derivative free optimization method for reservoir characterization inverse problem. EAGE - Conference \& Exhibition incorporating SPE EUROPEC, Jun 2010, Barcelone, Spain. hal-02284341

\section{HAL Id: hal-02284341 https://hal-ifp.archives-ouvertes.fr/hal-02284341}

Submitted on 11 Sep 2019

HAL is a multi-disciplinary open access archive for the deposit and dissemination of scientific research documents, whether they are published or not. The documents may come from teaching and research institutions in France or abroad, or from public or private research centers.
L'archive ouverte pluridisciplinaire HAL, est destinée au dépôt et à la diffusion de documents scientifiques de niveau recherche, publiés ou non, émanant des établissements d'enseignement et de recherche français ou étrangers, des laboratoires publics ou privés. 


\title{
A derivative free optimization method for reservoir characterization inverse problem
}

\author{
Hoël Langouët, Delphine Sinoquet and Sébastien Da Veiga
}

\begin{abstract}
These data (pressure, oil/water/gas rates at the wells and 4D seismic data) are compared with simulated data to determine petrophysical properties of the reservoir. The underlying optimization problem requires dedicated techniques : derivatives are often not available, the associated forward problems are CPU time consuming and some constraints may be introduced to handle a priori information.

In this paper, we propose a derivative free optimization method based on trust region approach coupled with local quadratic interpolating models of the cost function and of non linear constraints.

Results obtained with this method on a synthetic reservoir application with the joint inversion of production data and 4D seismic data are presented. Its performances are compared with a classical sequential quadratic programming method in terms of numbers of simulation of the forward problem.
\end{abstract}




\section{Introduction}

The goal of reservoir characterization is the estimation of unknown reservoir parameters by integrating available data in order to take decisions for production scheme and to predict the production of the field in the future. Reservoir parameters can be classified in two classes: those related to the geological modeling (spatial distribution of porosity, permeability, faults), and those related to the fluid flow modeling (relative permeability curves, productivity index of the wells). These parameters cannot be directly determined by measurements (or only locally using well logs). This is the reason why this parameter estimation problem is formulated as an inverse problem with some forward simulators that compute synthetic measurable data from the parameters : production data acquired at production/injection wells (e.g. bottom-hole pressure, gas-oil ratio, oil rate), time lapse seismic data (more precisely compressional and shear wave impedances for different seismic campaigns at different calendar times during the production of the field). The associated forward models consist of a fluid flow simulator and a petro-elastic model (PEM) based on rock physic Gassmann equations. For further details on this application see Roggero et al. (2008). Solving these forward problems is often CPU time consuming and does not provide the derivatives with respect to the parameters.

The underlying optimization problem is formulated as the minimization of a least-squares objective function composed of two terms, one for the production data mismatch and one for the seismic data mismatch. Some weights are introduced to account for data uncertainties and modeling errors. In practice, these problems are often solved by nonlinear optimization methods, as SQP method (Sinoquet and Delbos, 2008) with derivatives approximated by finite differences (FD).

In our application, the use of the classical Gauss-Newton algorithm is often infeasible because the computation of the Jacobian matrix is CPU time consuming and its storage is impossible for large datasets involving seismic data. Consequently, a natural alternative choice is a quasi-Newton approach based on classical BFGS approximation of the Hessian of the objective function with derivatives approximated by FD. Although these methods are particularly efficient for the determination of active constraints, the number of function evaluations is usually too high for industrial problems with expensive simulators. Furthermore, the choice of the FD step, crucial for the convergence of this method, is generally cumbersome because it depends on the accuracy of the function computation which is difficult to estimate in practice. This is the reason why, in this paper, we propose an adapted method based on local surrogate models (Conn et al., 2000, 2009; Powell, 2004): these methods are inspired by SQP methods with trust region globalization. The proposed Sequential Quadratic Approximation method (SQA) is an extension for constrained optimization of NEWUOA, the efficient Derivative Free Optimization method (DFO) of Powell (2004).

In the first part of this paper, we describe the main features of the SQA method. The second part is dedicated to a 4D synthetic reservoir application with the joint inversion of production data and 4D seismic data.

\section{SQA Method}

The SQA method is a DFO method adapted to constrained optimization of expensive nonlinear functions: $\min _{x \in \mathbb{R}^{n}} f(x)$ subject to $C_{N D}(x) \leq 0, C_{D}(x) \leq 0$, where the difficult constraints $C_{D}$ (responses of simulator with unknown derivatives) and the constraints with given derivatives $C_{N D}$ are separated $\left(C_{N D}: \mathbb{R}^{n} \rightarrow \mathbb{R}^{n_{N D}}, C_{D}: \mathbb{R}^{n} \rightarrow \mathbb{R}^{n_{D}}\right)$.

At an initial stage, a quadratic model $\tilde{f}$ of the function $f$ is constructed in a neighborhood of the current point. It interpolates $m$ points ${ }^{1}$ (usually $m=2 n+1$ is chosen) in the admissible domain (at least matching linear constraints). For each difficult constraint, a quadratic model $\tilde{C}_{D}$ is also constructed from the same interpolation points. Then, at each iteration, the current quadratic model $\tilde{f}$ is minimized under constraints $C_{N D}$ and models of difficult constraints $\tilde{C}_{D}$ in a trust region of radius $\Delta$ around the optimal point by a SQP method: $\min _{d} \tilde{f}\left(x_{o p t}+d\right)$ subject to $C_{N D}\left(x_{o p t}+d\right) \leq 0, \tilde{C}_{D}\left(x_{o p t}+d\right) \leq 0,\|d\| \leq \Delta$.

\footnotetext{
${ }^{1}$ The incomplete quadratic models $(m<(n+1)(n+2) / 2)$ are completed by minimizing the Frobenius norm of the hessian matrix variations of the models (Powell, 2004)
} 
The function $f$ is evaluated at $x^{*}$ the solution of this minimization: if the merit function, measuring the constraint violation and $f$, has decreased, $x^{*}$ is validated as the current optimal point. Otherwise another point is added in order to improve the quadratic models: a criterion based on the interpolating Lagrange polynomial is maximized, see Powell (2004). Finally, the trust region radius $\Delta$ is updated according to the comparison of the reduction of the merit function based on models $\tilde{f}$ and $\tilde{C}_{D}$ and the effective reduction of the merit function based on $f$ and $C_{D}$. So, $\Delta$ is increased if the models have the correct trends compared to $f$ and $C_{D}$ or decreased otherwise. The quadratic models $\tilde{f}$ and $\tilde{C}_{D}$ are updated to interpolate the $m$ closest points (where $f$ and $C_{D}$ were evaluated) to the current optimal point. The algorithm is stopped when the minimal trust region radius (accuracy on the solution requested by the user) is reached or when the maximum number of simulations given by the user is achieved.

\section{Reservoir application: inversion of monitor seismic datasets}

The PUNQ test case is a 3D synthetic reservoir model derived from real field data. It was already used for comparative inversion studies in the European PUNQ project (Floris, 2001) and for validation of constrained modeling and optimization scheme development methods (Roggero, 2001). The top structure of the reservoir is presented in Figure 1 (Top left). The reservoir is surrounded by an aquifer in the north and the west, and delimited by a fault in the south and the east. A small gas cap is initially present. The geological model is composed of five independent layers. The layers 1, 3, 4 and 5 are assumed to be of good quality, while the layer 2 is of poorer quality.

The initial model consists of a $19 \times 28 \times 5$ grid, with a constant step of $180 \mathrm{~m}$ in the horizontal $X$ and $Y$ directions. In order to illustrate the potential of the algorithm on large seismic data sets, we decided to build a larger model. Therefore, our reference model consists of a $76 \times 56 \times 5$ grid, with a constant step of $45 \mathrm{~m}$ in the $X$ direction and $90 \mathrm{~m}$ in the $Y$ direction. Each geological unit is modeled by one layer, with a Gaussian distribution of the porosities and a spherical variogram. The geostatistical simulation parameters are listed in Table of Figure 1. The permeability on each layer is defined by a $(\log K-\phi)$ relationship, i.e. $\log \left(K_{x}\right)=A \phi+B$ with constant ratios $K_{y} / K_{x}$ and $K_{z} / K_{x}$. The corresponding reference parameters are given in Table of Figure 1. Then, the geostatistical simulations are upscaled to come back to the original $19 \times 28 \times 5$ grid in order to work with a faster fluid flow simulation in the reservoir. The synthetic production data are produced by a numerical simulation using the ATHOS model over a eight-year period. The production results selected as synthetic measurements are the gas oil ratio (GOR), the bottomhole pressure (BHP) and the water cut value (WCUT) at the six producing wells (PRO-1, 4, 5, 11, 12 and 15). We give in the middle of Figure 1 the reference production data for all wells. Then, pressure and saturations maps simulated by the ATHOS model at times 0 days, 181 days (half a year) and 2192 days (six years) are extracted and downscaled to the $76 \times 56 \times 5$ grid. A petro-elastic model (PEM) involving Gassmann and Hertz equations is defined with given bulk and dry modulus, bulk densities and Hertz exponents. The combination of downscaled pressure and saturations maps with this petroelastic model allows to compute synthetic P impedance maps at times 0, 181 and 2192 days. These maps serve as a synthetic 4D seismic data set. An example of the reference impedance map on layer 5 at time 0 and Delta impedance maps at times 181 and 2192 is depicted in the bottom of Figure 1.

For history matching, the parameters of the simulation model are constrained by both production and seismic data. An optimal matching is sought by minimization of an objective function defined as follows: $f(x)=\sum_{i=1}^{6} \sum_{j=1}^{n_{p}}\left(d_{P_{i}}^{\text {sim }}\left(x, t_{j}\right)-d_{P_{i}}^{\text {obs }}\left(t_{j}\right)\right)^{2}+\sum_{k=1}^{n_{\text {grid }}}\left(d_{S}^{\text {sim }}(x, k)-d_{S}^{\text {obs }}(k)\right)^{2}$, where $i=1, \ldots, 6$ is the well index, $t_{j}, j=1, \ldots, n_{p}$ are the measurement times of production data and $k=1, \ldots, n_{\text {grid }}$ denotes the cell indices. The geometric data, the geological structure, the fluid properties and the geomechanical parameters of the PEM are presumed known. The inversion parameters are the porosity means (one for each layer), the A and B coefficients for the permeabilities (two coefficients per layer) and the permeability ratios (two ratios per layer). 


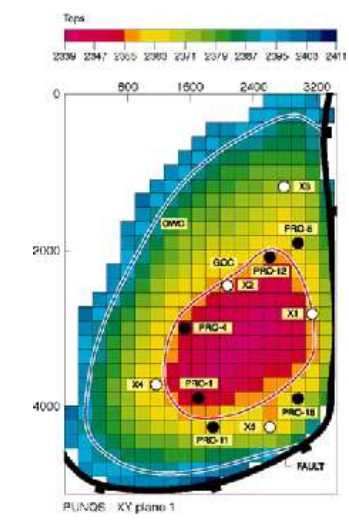

Corresponding reference of geostatistical simulation parameters

\begin{tabular}{|c|c|c|c|c|c|c|}
\hline & $\phi$ mean & $\phi$ variance & $A$ & $B$ & $K y / K x$ & $K z / K x$ \\
\hline Layer 1 & 0.1722 & 0.0078 & 8.585 & 0.701 & 1 & 0.364 \\
\hline Layer 2 & 0.0802 & 0.0004 & 14.383 & 0.258 & 1 & 0.339 \\
\hline Layer 3 & 0.1677 & 0.0050 & 8.683 & 0.781 & 1 & 0.314 \\
\hline Layer 4 & 0.1615 & 0.0006 & 4.209 & 1.789 & 1 & 0.211 \\
\hline Layer 5 & 0.1892 & 0.0049 & 8.98 & 0.793 & 1 & 0.296 \\
\hline
\end{tabular}

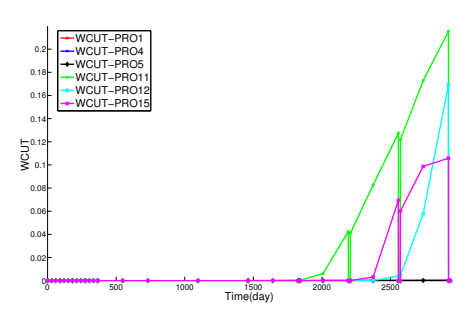

(a) WCUT

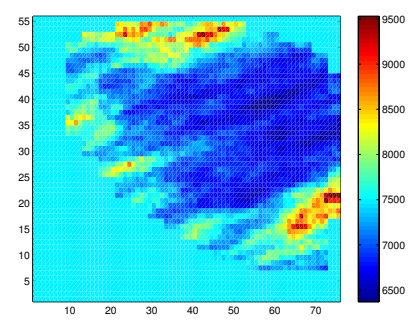

(d) $I_{P}$ at time 0

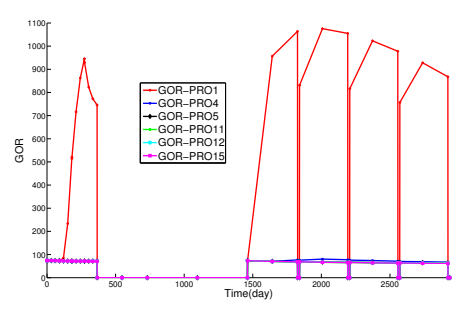

(b) GOR

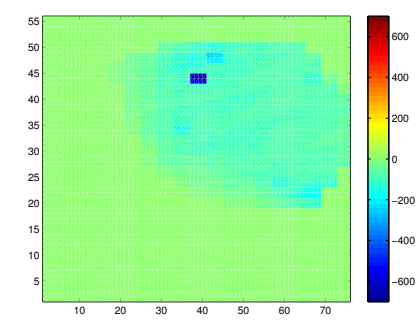

(e) $\Delta I_{P}$ at time 181

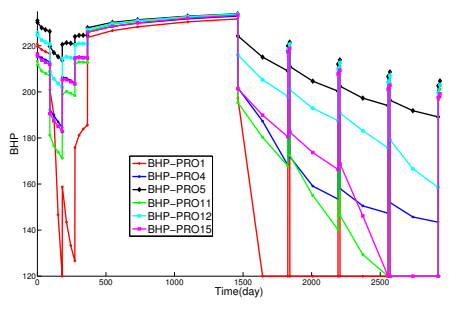

(c) BHP

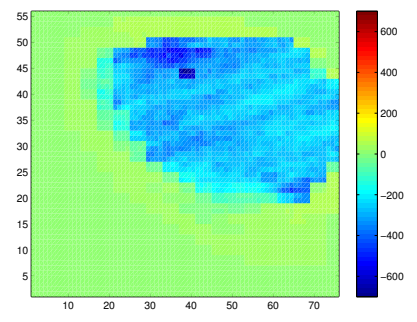

(f) $\Delta I_{P}$ at time 2192

Figure 1: PUNQ field: Reference production data (middle), seismic data (P impedance map) for layer 5 (bottom).

The SQPAL BFGS method ((Sinoquet and Delbos, 2008)) and the SQA method are used to minimize the objective function with respect to these 25 inversion parameters. The results obtained by both methods are presented in the top of Figure 2, where the evolution of the objective function is depicted with respect to the number of fluid flow simulations. In both cases, we can observe that the objective function decreases: the two different methods seem to converge to a local minimum. We can also not that SQA requires more evaluations during the initialization phase than SQPAL ( $m=2 n+1$ compared to FD computations which require $n+1$ ). However, SQA achieves a greater reduction of the objective function much faster than SQPAL. This is the key advantage of SQA, since it allows to reduce the number of evaluations of the simulator, which are very CPU time consuming. The production data at well 12 obtained by the initial model and the SQA matched model are compared to the reference data in the middle of Figure 2. SQA provides a model which is much closer to the reference production data than the initial model. The reference, initial, and optimal impedance map of layer 5 at time 0 obtained with the SQA method is given in Figure 2. We can observe that SQA is successful to give a good match for the seismic data.

\section{Conclusions}

SQA method gives promising results on a 4D reservoir characterization problem: the results obtained show that this DFO method decreases the objective function faster than the classical optimization method 
based on BFGS approach. A study to take into account nonlinear constraints (with unknown derivatives from the simulator) is in progress in order to solve another application in reservoir characterization. This study aims at computing uncertainty estimations and confidence intervals on the solutions of history matching problems.

\section{References}

Conn, A., Gould, N. and Toint, P. [2000] Trust-Region Methods. MPS-SIAM Series on Optimization.

Conn, A., Scheinberg, K. and Vicente, L.N. [2009] Introduction to Derivative-Free Optimization. MPS-SIAM Series on Optimization.

Floris [2001] Methods for quantifying the uncertainty of production forecasts - a comparative study, vol. 7. published in J.Petroleum Geoscience.

Powell, M. [2004] The NEWUOA software for unconstrained optimization without derivatives.

Roggero [26-28 ${ }^{\text {th }}$ June 2001] Constraining stochastic reservoir models to dynamic data: an integrated approach. Rueil, France, OAPEC seminar.

Roggero, F. et al. [29 June 3 July 2008] Constraining Reservoir Models to Production and 4D Seismic Data Application to the Girassol Field. Madrid, Offshore Angola, 19th World Petroleum Congress.

Sinoquet, D. and Delbos, F. [8 - 11 September 2008] Adapted nonlinear optimization method for production data and $4 D$ seismic inversion. Bergen, Norway, $11^{\text {th }}$ ECMOR.

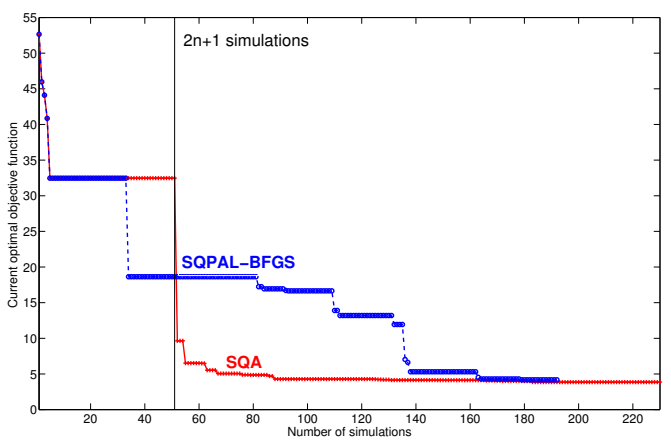

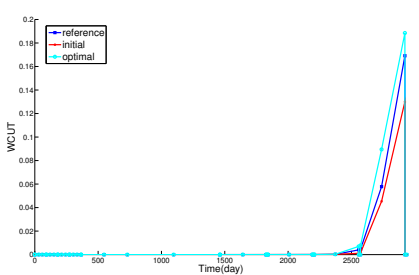

(a) WCUT for well 12

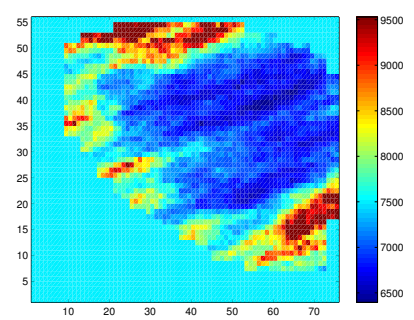

(d) Reference

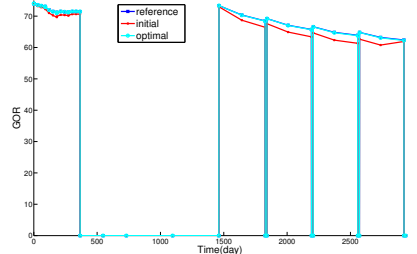

(b) GOR for well 12

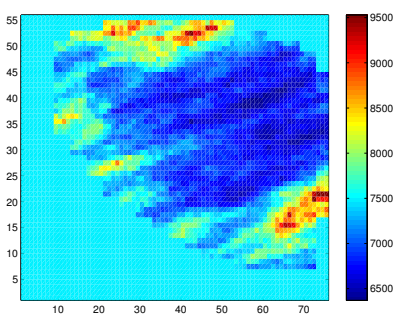

(e) Initial

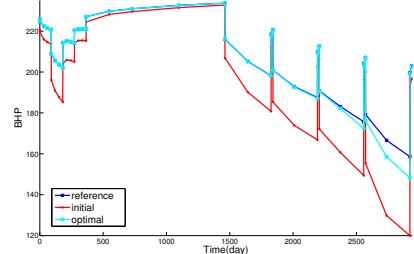

(c) BHP for well 12

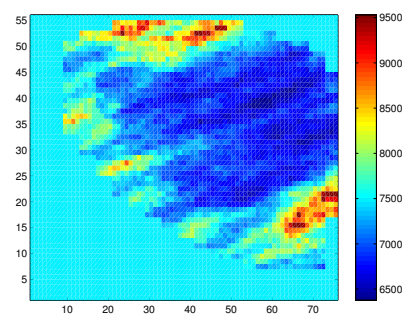

(f) Optimal

Figure 2: Top: Objective function versus simulation numbers for two optimization runs, respectively with SQA (solid line, red cross), and with BFGS method with line search globalization (dashed line, blue circle). Middle: Comparison between reference production data (blue) and simulated production data for initial point (red) and optimal point obtained with SQA (cyan). Bottom: P Impedance maps at time 0 obtained with SQA. 